Pacific Journal of Mathematics

A CLASS OF ABELIAN GROUPS CLOSED UNDER DIRECt 


\title{
A CLASS OF ABELIAN GROUPS CLOSED UNDER DIRECT LIMITS AND SUBGROUPS FORMATION
}

\section{ChIN-Shui Hsü}

\begin{abstract}
This is a solution to the following problem: Which classes of Abelian groups are closed under taking subgroups and direct limits. (Problem 6(a), L. Fuchs, Infinite Abelian Groups, $I, A-P$.) Each such class is uniquely determined by its subclass of finitely generated Abelian groups, which in turn can be described by a set of numerical invariants.

In like manner, an analogue for modules over a Dedekind domain is also obtained.
\end{abstract}

Throughout this paper, we shall make no distinction between isomorphic copies of the same Abelian group. Thus when we say that an Abelian group belongs to a class we mean that the former is isomorphic to a member of the latter.

In many places, proofs of our results are omitted. This is because our discussion is of a constructive nature. As soon as a construction is carried out, the fact that it has the desired property becomes selfevident. Thus a formal proof is not necessary.

2. A reduction of the problem. Suppose $\Gamma$ is a class of Abelian groups satisfying:

(I) $A \in \Gamma$, and $B$ is a subgroup of $A$, implies $B \in \Gamma$.

(II) If $\left\{A_{\alpha}, \pi_{\alpha \beta}\right\}$ is a direct system with $A_{\alpha} \in \Gamma$, then $\left(\lim A_{\alpha}\right) \in \Gamma$. Define $\Gamma_{0}$ to be the subclass of finitely generated Abelian groups in $\Gamma$. Suppose $\Phi$ is a class of finitely generated Abelian groups satisfying (I), define $\widehat{\Phi}$ as follows: $A \in \widehat{\Phi}$ if there is a direct system $\left\{A_{\alpha}, \pi_{\alpha \beta}\right\}$ s.t.

$$
\begin{aligned}
& A_{\alpha} \in \Phi \\
& \pi_{\alpha \beta} \text { are monomorphisms } \\
& \lim A_{\alpha}=A
\end{aligned}
$$

(i.e., an Abelian group $A \in \widehat{\Phi}$ if it is the union of a directed (by inclusions) family of finitely generated subgroups each of which belongs to $\Phi)$.

Lemma 2.1. If $\left\{A_{\alpha}, \pi_{\alpha \beta}\right\}$ is a direct system s.t. $A_{\alpha} \in \Phi$, then $\left(\lim A_{\alpha}\right) \in \hat{\Phi}$.

Proof. For a fixed $\alpha$, among the subgroups $\left(\operatorname{Ker} \pi_{\alpha \beta}\right)$ of $A_{\alpha},(\beta \geqq$ $\alpha$ ), there is a maximum one, (because $A_{\alpha}$ is finitely generated). The- 
refore, there is $\alpha^{\prime} \geqq \alpha$ s.t. $\left(\operatorname{Ker} \pi_{\alpha \alpha^{\prime}}\right)=\left(\operatorname{Ker} \pi_{\alpha \beta}\right) \forall \beta \geqq \alpha$. Let $A_{\alpha}^{\prime}=$ $A_{\alpha} /\left(\operatorname{Ker} \pi_{\alpha \alpha^{\prime}}\right)$, then for $\gamma \geqq \alpha, \pi_{\alpha \gamma}: A_{\alpha} \rightarrow A_{\gamma}$ induces a monomorphism $\pi_{\alpha \gamma}^{\prime}: A_{\alpha}^{\prime} \rightarrow A_{\gamma}^{\prime}$. Obviously $\left\{A_{\alpha}^{\prime}, \pi_{\alpha \gamma}^{\prime}\right\}$ is a direct system and $\left(\lim A_{\alpha}^{\prime}\right)=$ $\left(\lim _{\alpha} A_{\alpha}\right)$. Therefore, $\left(\stackrel{\lim }{\longrightarrow} A_{\alpha}\right) \in \hat{\Phi}$.

THEOREM 2.2. If $\Gamma$ is a class of Abelian groups satisfying (I), (II), then

(i) $\Gamma_{0}$ satisfies (I),

(ii) $\left(\hat{\Gamma}_{0}\right)=\Gamma$.

If $\Phi$ is a class of finitely generated Abelian groups satisfying (I), then

(iii) $\hat{\Phi}$ satisfies (I), (II),

(iv) $(\widehat{\Phi})_{0}=\Phi$.

Proof. (i) is obvious.

(ii) If $A \in \Gamma$, let $\left\{A_{\alpha}\right\}$ be the family of all finitely generated subgroups of $A$, then each $A_{\alpha} \in \Gamma_{0}$. Since $A=\bigcup A_{\alpha}, A \in\left(\Gamma_{0}\right)$.

(iii) $\hat{\Phi}$ satisfies (I): Suppose $A \in \hat{\Phi}$, and $B$ is a subgroup of $A$. We have a direct family $\left\{A_{\alpha}\right\}$ of subgroups of $A$ s.t.

$$
\begin{aligned}
& A_{\alpha} \in \Phi, \\
A= & \bigcup A_{\alpha} .
\end{aligned}
$$

Let $B_{\alpha}=B \cap A_{\alpha}$, then

$$
\begin{aligned}
& B_{\alpha} \in \Phi, \\
B= & \bigcup B_{\alpha} .
\end{aligned}
$$

Hence $B \in \hat{\Phi}$.

$\hat{\Phi}$ satisfies (II): Suppose we have a direct system $\left\{A_{\alpha}, \pi_{\alpha \beta}\right\}_{\alpha, \beta \in A}$ s.t.

$$
\begin{aligned}
& A_{\alpha} \in \hat{\Phi}, \\
A= & \stackrel{\lim }{\longrightarrow} A_{\alpha} .
\end{aligned}
$$

For each $\alpha$, we have a directed family $\left\{A_{\alpha, a}\right\}_{a \in \Lambda_{\alpha}}$ of finitely generated subgroups of $A_{\alpha}$ s.t.

$$
\begin{aligned}
& A_{\alpha, a} \in \Phi, \\
A_{\alpha}= & \bigcup A_{\alpha, a} .
\end{aligned}
$$

Let $\hat{\Lambda}=\left\{(\alpha, a) \mid a \in \Lambda_{\alpha}\right\}$ and define $(\alpha, a) \leqq(\beta, b)$ if

$$
\begin{gathered}
\alpha \leqq \beta, \\
\pi_{\alpha \beta}\left(A_{\alpha, a}\right) \leqq A_{\beta, b} .
\end{gathered}
$$

We claim that $\tilde{\Lambda}$ is a directed set: $\operatorname{Given}(\alpha, a),(\beta, b) \in \tilde{\Lambda}$, there 


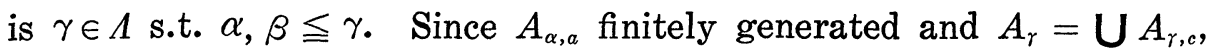
there is $c^{\prime} \in \Lambda_{\gamma}$ s.t. $\pi_{\alpha \gamma}\left(A_{\alpha, a}\right) \subseteq A_{\gamma, c^{\prime}}$. Likewise there is $c^{\prime \prime} \in \Lambda_{\gamma}$ s.t. $\pi_{\beta \gamma}\left(A_{\beta, b}\right) \leqq A_{\gamma, c^{\prime \prime}}$. Choose $c \in \Lambda_{\gamma}$ s.t. $c^{\prime}, c^{\prime \prime} \leqq c$, then $(\alpha, a),(\beta, b) \leqq(\gamma, c)$.

For $(\alpha, a) \leqq(\beta, b)$, define

$$
\pi_{(\alpha, a),(\beta, b)}: A_{\alpha, a} \longrightarrow A_{\beta, b}
$$

by $\pi_{(\alpha, a),(\beta, b)}(x)=\pi_{\alpha \beta}(x) \forall x \in A_{\alpha, a}$, then

$$
\left\{A_{\alpha, a}, \pi_{(\alpha, a),(\beta, b)}\right\}
$$

is a direct system with

$$
\stackrel{\lim }{\longrightarrow} A_{\alpha, a}=A
$$

Therefore, $A \in \widehat{\Phi}$.

(iv) If $A \in(\widehat{\Phi})_{0}$, then there is a directed family $\left\{A_{\alpha}\right\}$ of subgroups of $A$ s.t.

$$
\begin{aligned}
& A_{\alpha} \in \Phi, \\
A=\bigcup & \bigcup A_{\alpha} .
\end{aligned}
$$

Since $A$ is finitely generated, $A=A_{\alpha}$ for some $\alpha$. Therefore $A \in \Phi$.

3. A further reduction. Let $\Sigma$ be the class of all finitely generated Abelian groups. For $A, B \in \Sigma, A \leqq B$ means $A$ is (isomorphic to) a subgroup of $A$. Clearly this is a partial ordering on $\Sigma$. A subclass $\Theta$ of $\Sigma$ is called an ideal if it satisfies (I) and

(III) $A, B \in \Theta$ implies there is $C \in \Theta$ s.t. $A, B \leqq C$.

LEMMA 3.1. The union of a directed (by inclusions) family of ideals is an ideal. In particular the union of a linearly ordered family of ideals is an ideal.

The proof is obvious.

Suppose $\Phi$ is a class of finitely generated Abelian groups satisfying (I), we use $\Phi_{m}$ to denote the family of all maximum ideals in $\Phi$, (i.e., those which are maximum among ideals contained in $\Phi$ ). Suppose $\Delta$ is a family of ideals. We say that $\Delta$ is irredundant if none of its members is contained in another member, (i.e., each member is maximum in the family). We say that $\Delta$ is closed if given an ideal $\Theta^{\prime} \leqq$ $\mathrm{U}_{\theta \in \Delta} \Theta$ there is $\Theta^{\prime \prime} \in \Delta$ s.t. $\Theta^{\prime} \subseteq \Theta^{\prime \prime}$. We define $\Phi(\Delta)=\mathbf{U}_{\theta \in \Delta} \Theta$.

THOREM 3.2. If $\Phi$ is a class of finitely generated Abelian groups satisfying (I), then

(i ) $\Phi=\Phi\left(\Phi_{m}\right)$, 
(ii) $\Phi_{m}$ is irredundant,

(iii) $\Phi_{m}$ is closed.

If $\Delta$ is a closed irredundant family of ideals, then

(iv) $\Phi(\Delta)$ satisfies (I),

(v) $(\Phi(\Delta))_{m}=\Delta$.

Proof. (i) and (iii) are obtained through a routine use of Zorn's Lemma.

(ii) and (iv) are obvious.

(v) is a consequence of the definition of closeness.

THEOREM 3.3. If $\Delta$ is a closed, irredundant family of ideals, and $\Phi=\Phi(\Delta)$, then

$$
\hat{\Phi}=\mathbf{U}_{\theta \in \Delta} \hat{\Theta} .
$$

Proof. Clearly $\hat{\Phi} \supseteqq \mathrm{U}_{\theta \in \Delta} \hat{\Theta}$.

If $A \in \widehat{\Phi}$, then there is a directed family $\left\{A_{\alpha}\right\}$ of finitely generated subgroups of $A$ s.t.

$$
\begin{aligned}
& A_{\alpha} \in \Phi, \\
& A=\bigcup A_{\alpha} \text {. }
\end{aligned}
$$

Define $\Phi_{A}$ as follows: $B \in \Phi_{A}$ if $B \leqq A_{\alpha}$ for some $\alpha$. Clearly $\Phi_{A}$ is an ideal contained in $\Phi$. Since $\Delta$ is closed, there is $\Theta \in \Delta$ s.t. $\Phi_{A} \subseteq$ $\Theta$. Clearly $A \in \hat{\Theta}$.

Therefore, $\hat{\Phi}=\bigcup_{\theta \in \Delta} \hat{\Theta}$.

\section{Arithmetization. Let}

$\mathscr{P}=$ the set consisting of 0 and all positive integral powers of every prime number,

$\mathscr{N}=$ the set of all nonnegative integers,

$\hat{\mathscr{N}}=\mathscr{N} \cup\{\infty\}$,

$\Omega=$ the set of all mappings $\mu: \mathscr{P} \rightarrow \hat{\mathscr{N}}$ satisfying the condition: $\mu\left(p^{m}\right) \geqq \mu\left(p^{n}\right)$ whenever $m \leqq n,(\hat{\mathscr{N}}$ is ordered in the obvious manner), $\Omega_{0}=$ the set of all $\mu \in \Omega$ satisfying the conditions: (i) $\mu(x) \neq \infty \forall x \in$ $\mathscr{P}$; (ii) $\mu(x)=0$ for almost all $x \in \mathscr{P}$.

For $\lambda, \mu \in \Omega, \lambda \leqq \mu$ means $\lambda(x) \leqq \mu(x) \forall x \in \mathscr{P}$. Obviously this is a partial ordering on $\Omega$. Define $\chi: \Sigma \rightarrow \Omega_{0}$ as follows: For $A \in \Sigma, A=$ $A_{1} \oplus \cdots \oplus A_{s}$, where each $A_{j}$ is isomorphic with $Z / x Z$ for some $x \in$ $\mathscr{P}$. Set

$$
\chi(A)(x)=\text { the number of } A_{j} \geqq Z / x Z \text {. }
$$

Clearly $\chi$ is an order isomorphism. Suppose that $\Theta$ is an ideal in $\Sigma$. 
Define $\chi(\Theta) \in \Omega$ as follows:

$$
\chi(\Theta)(x)=\text { l.u.b. }\{\chi(A)(x) \mid A \in \Phi\} .
$$

For $\mu \in \Omega$, define

$$
\Phi(\mu)=\{A \in \Sigma \mid \chi(A) \leqq \mu\} .
$$

Suppose that $\Delta^{\prime}$ is a subset of $\Omega$. We say that $\Delta^{\prime}$ is irredundant if every element of $\Delta^{\prime}$ is maximum in $\Delta^{\prime}$. We say that $\Delta^{\prime}$ is closed if the following condition is satisfied: Given $\omega \in \Omega$ with the property that for each $\mu \in \Omega_{0}$, s.t. $\mu \leqq \omega$, there is $\delta^{\prime} \in \Delta^{\prime}$ s.t. $\mu \leqq \delta^{\prime}$, then there is $\delta \in \Delta^{\prime}$ s.t. $\omega \leqq \delta$.

Lemma 4.1. If $\Theta$ is an ideal in $\Sigma$ and $A \in \Sigma$ s.t. $\chi(A) \leqq \chi(\Theta)$, then $A \in \Theta$.

Proof. Since $A \in \Sigma$, there are $x_{1}, \cdots, x_{s} \in \mathscr{P}$ s.t. $\chi(A)(x)=0$ except $x=x_{1}, \cdots, x_{s}$. Since $\chi(A) \leqq \chi(\Theta)$, for each $x_{j}$, there is $A_{j} \in \Theta$ s.t.

$$
\chi(A)\left(x_{j}\right) \leqq \chi\left(A_{j}\right)\left(x_{j}\right) \text {. }
$$

Since $\Theta$ is an ideal, there is $A^{\prime} \in \Theta$ s.t.

$$
A_{1}, A_{2}, \cdots \leqq A^{\prime} \text {. }
$$

Obviously $\chi(A) \leqq \chi\left(A^{\prime}\right)$, i.e., $A \leqq A^{\prime}$, and hence $A \in \Theta$.

CoROLlary 4.2. Given two ideals $\Theta, \Theta^{\prime}, \chi(\Theta)=\chi\left(\Theta^{\prime}\right)$ iff $\Theta=\Theta^{\prime}$.

Lemma 4.3. For $\mu \in \Omega$,

(i) $\Phi(\mu)$ is an ideal,

(ii) $\chi(\Phi(\mu))=\mu$.

Proof. (i) $\Phi(\mu)$ satisfies (I) is obvious.

$\Phi(\mu)$ satisfies (III): Suppose $A, B \in \Phi(\mu)$. Define $\lambda: \mathscr{P} \rightarrow \mathscr{N}^{\circ}$ by

$$
\lambda(x)=\max \{\chi(A)(x), \chi(B)(x)\}
$$

$\forall x \in \mathscr{P}$. Obviously $\lambda \in \Omega_{0}$, and $\lambda \leqq \mu$. Since $\chi$ is an isomorphism, there is $C \in \Sigma$ s.t. $\chi(C)=\lambda$. Obviously $A, B \leqq C$, and $C \in \Phi(\mu)$.

(ii) According to the definition, for $x \in \mathscr{P}, A \in \Phi(\mu)$, we have $\chi(A)(x) \leqq \mu(x)$. Therefore, $\chi(\Phi(\mu)) \leqq \mu$.

If $\chi(\Phi(\mu)) \neq \mu$, then there is $x \in \mathscr{P}$ s.t. $\chi(\Phi(\mu))(x)<\mu(x)$. Suppose $\chi(\Phi(\mu))(x)=n$. (We cannot have $\chi(\Phi(\mu))(x)=\infty$ because $\infty<\mu(x)$ does not hold.) Let $A=(Z / x Z) \oplus \cdots \oplus(Z / x Z),(n+1$ copies $)$, then $\chi(A)(x)=n+1 \leqq \mu(x), \chi(A)(z)=0 \leqq \mu(z) \forall z \neq x$. Therefore, $A \in \Phi(\mu)$. This contradicts the assumption that $\chi(\Phi(\mu))(x)=n$. Hence $\chi(\Phi(\mu))=\mu$. 
THEOREM 4.4. If $\Delta$ is a closed irredundant family of ideals in $\Sigma$, then $\{\chi(\Theta) \mid \Theta \in \Delta\}$ is

(i) closed,

(ii) irredundant.

If $\Delta^{\prime}$ is a closed irredundant subset of $\Omega$, then $\left\{\Phi(\mu) \mid \mu \in \Delta^{\prime}\right\}$ is

(iii) closed,

(iv) irredundant.

The proof is obvious.

Combining all the earlier results together we have

THEOREM 4.5. (i) If $\Delta^{\prime}$ is a closed, irredundant subset of $\Omega$, then

$$
\bigcup_{\mu \in \Delta^{\prime}} \widehat{\Phi(\mu)}
$$

is a class of Abelian group satisfying (I) and (II).

(ii) If $\Gamma$ is a class of Abelian groups satisfying (I) and (II), then

$$
\Gamma=\bigcup_{\mu \in \Delta^{\prime}} \widehat{\Phi(\mu)}
$$

where $\Delta^{\prime}=\left\{\chi(\Theta) \mid \Theta \in\left(\Gamma_{0}\right)_{m}\right\}$.

5. An explicit construction. We shall adopt the following notations:

$T(A), A$ is an Abelian group: The torsion part of $A$.

$T_{0}(A): A / T(A)$.

$T_{p}(A), p$ is a prime number: The $p$-primary component of $T(A)$.

rank $(A)$ : the number of summands in the direct sum decomposition of the injective envelope of $A$ into indecomposable subgroups.

THEOREM 5.1. For $\mu \in \Omega, \widehat{\Phi(\mu)}$ consists of Abelian groups A subject to the following conditions:

(i) If $\mu(0)=\infty$, then $T_{0}(A)$ can be arbitrary.

If $\mu(0) \neq \infty$, then $\operatorname{rank}\left(T_{0}(A)\right) \leqq \mu(0)$.

(ii) For each $p^{k} \in \mathscr{P}$, if $\mu\left(p^{k}\right)=\infty$, then $p^{k-1} T_{p}(A)$ can be arbitrary. If $\mu\left(p^{k}\right) \neq \infty$, then $\operatorname{rank}\left(p^{k-1} T_{p}(A)\right) \leqq \mu\left(p^{k}\right)$.

Proof. This is obvious. (Observe that these conditions are preserved under taking subgroups $T_{0}(), T_{p}()$ and direct limits.)

REMARK 5.2. In view of Theorem 4.5, there can be classes of Abelian groups satisfying (I) and (II) whose structures are extremely 
complicated. However, almost all known examples take the simplest possible form, viz., they are $\widehat{\Phi(\mu)}$ for certain $\mu \in \Omega$, e.g.,

(1) The class of all ( $p$-primary) co-cyclic groups is given by

$$
\mu(x)= \begin{cases}1, & x=p^{k}, k=1,2, \cdots \\ 0, & \text { otherwise. }\end{cases}
$$

(2) The class of all locally cyclic groups is givin by $\hat{\Phi}(\mu) \cup \hat{\Phi}(\nu), \nu(x)=\left\{\begin{array}{ll}1, & x=0, \\ 0, & x \neq 0,\end{array} \quad \nu(x)= \begin{cases}0, & x=0 \\ 1, & x \neq 0 .\end{cases}\right.$

(3) The class of all torsion groups is given by

$$
\mu(x)= \begin{cases}0, & x=0, \\ \infty, & x=0 .\end{cases}
$$

(4) The class of all torsion-free groups is given by

$$
\mu(x)= \begin{cases}\infty, & x=0, \\ 0, & x \neq 0 .\end{cases}
$$

(5) The class of all groups is given by

$$
\mu(x)=\infty \forall x \in \mathscr{P} \text {. }
$$

(6) The class of all groups annihilated by $n$ is given by

$$
\mu(x)= \begin{cases}\infty, & x \mid n, \\ 0, & x \nmid n\end{cases}
$$

REMARK 5.3. $\widehat{\Phi(\mu)}$ generalizes the class of all subgroups in a given Abelian group. In fact, for a fixed cardinal number $\boldsymbol{\aleph}$, the members of $\widehat{\Phi(\mu)}$ with cardinality $\leqq \aleph$ are exactly the subgroups in the direct sum constructed as follows:

(a) For each prime number $p$, if

$$
\begin{gathered}
\mu(p)=\cdots=\mu\left(p^{k_{1}}\right)=\lambda_{1} \\
<\mu\left(p^{k_{1}+1}\right)=\cdots=\mu\left(p^{k_{2}}\right)=\lambda_{2} \\
\vdots \\
<\mu\left(p^{k_{r}+1}\right)=\cdots=\lambda_{r+1}
\end{gathered}
$$

where $k_{1}<k_{2}<\cdots<k_{r}$, (including $r=0$ to mean $\mu(p)=\cdots=\lambda_{1}$ ), we put in the following summands:

$$
\begin{aligned}
& \lambda_{r+1} \text { copies of } Z\left(p^{\infty}\right), \\
& \left(\lambda_{j}-\lambda_{j+1}\right) \text { copies of } Z / p^{k_{j}} Z, j=2, \cdots, r, \\
& \left\{\begin{array}{l}
\lambda_{1}-\lambda_{2} \text { copies of } Z / p^{k_{1}} Z,\left(\lambda_{1}<\infty\right), \text { or } \\
\boldsymbol{\aleph} \text { copies } Z / p^{k_{1}} Z,\left(\lambda_{1}=\infty\right),
\end{array}\right.
\end{aligned}
$$


(in case $r=0, \lambda_{1}=\infty$ these are $\aleph$ copies of $Z\left(p^{\infty}\right)$ ).

(b) We also put in the following summands:

$$
\left\{\begin{array}{l}
\mu(0) \text { copies of } Q,(\mu(0)<\infty), \text { or } \\
\checkmark \text { copies of } Q,(\mu(0)=\infty) .
\end{array}\right.
$$

REMARK 5.4. One may wish that a class satisfying (I), (II) can be expressed either as a union of a finite number of $\widehat{\Phi(\mu)}$ or as a family of such which are mutually disjoined. The following example shows that this is not always possible.

Let $p_{1}, p_{2}, \cdots$ be a set of prime numbers and $\mu_{1}, \mu_{2}, \cdots \in \Omega$ be given by

$$
\mu_{i}\left(p_{j}^{k}\right)= \begin{cases}1, & j=i \quad \text { or } \quad k=1 \\ 0, & j \neq i, k>1\end{cases}
$$

Obviously $\Gamma=\mathrm{U} \widehat{\Phi\left(\mu_{i}\right)}$ satisfies (I), (II), and cannot be decomposed into finite or disjoined union in the above mentioned way.

REMARK 5.5. The definition of closed irredundant subsets of $\Omega$ is closely related to the concept of closed sets in a topological space. For an irredundant subset $\Delta$ of $\Omega$, let

$$
\Delta^{\prime}=\{\omega \in \Omega \mid \omega \leqq \delta \text { for some } \delta \in \Delta\},
$$

then $\Delta$ is closed iff the least upper bound of every net in $\Delta^{\prime}$ is still in $\Delta^{\prime}$.

6. A generalization. The results in the previous sections can be extended to modules over a Dedekind domain. (For basic properties of a Dedekind domain we refer to [2].) This is carried out in the following. Proofs are omitted because they are essentially the same as the case of Abelian groups. We adopt the following notations:

$R$ : A Dedekind domain.

$\mathscr{P}$ : The set of all primary ideals in $R$, (i.e., 0 , and powers of nonzero prime ideals).

$\mathscr{N}:$ The set of all nonnegative integers.

$\hat{\mathscr{N}}: \hat{N} \cup\{\infty\}$.

$\Omega$ : The set of all mappings $\mu: \mathscr{P} \rightarrow \mathscr{N}$ satisfying $\mu(x) \geqq \mu(y)$ whenever $x \mid y, x, y \neq 0$.

$\Omega_{0}$ : The set of $\mu \in \Omega$ satisfying: (i) $\mu(x) \neq \infty$, (ii) $\mu(x)=0$ for almost all $x \in \mathscr{P}$.

$T(A),(A$ is an $R$-module): The torsion part of $A$.

$T_{0}(A): \quad A / T(A)$.

$T_{p}(A),(p$ is a prime ideal of $R)$ : The $p$-primary component of 
$T(A)$.

rank $(A)$ : The number of summands in the direct sum decomposition of the injective envelope of $A$ into indecomposable $R$-modules.

For $\lambda, \mu \in \Omega, \lambda \leqq \mu$ means that $\lambda(x) \leqq \mu(x) \forall x \in \mathscr{P}$. A subset $\Delta^{\prime}$ of $\Omega$ is irredundant if every element of $\Delta^{\prime}$ is maximum in $\Delta^{\prime}$. It is closed if the following condition is satisfied: Given $\omega \in \Omega$ with the property that for $\mu \in \Omega_{0}, \mu \leqq \omega$ implies there is $\delta^{\prime} \in \Delta^{\prime}$ s.t. $\mu \leqq \delta^{\prime}$, then there is $\delta \in \Delta^{\prime}$ s.t. $\omega \leqq \delta$.

For $\mu \in \Omega$, define $\widehat{\Phi(\mu)}$ as the class of all $R$-modules subject to the following conditions:

(i) If $\mu(0)=\infty, T_{0}(A)$ can be arbitrary.

If $\mu(0) \neq \infty, \operatorname{rank}\left(T_{0}(A)\right) \leqq \mu(0)$.

(ii) If $\mu\left(p^{k}\right)=\infty$, ( $p$ is a prime ideal of $\left.R\right), p^{k-1} T_{p}(A)$ can be arbitrary.

If $\mu\left(p^{k}\right) \neq \infty, \operatorname{rank}\left(p^{k-1} T_{p}(A)\right) \leqq \mu\left(p^{k}\right)$.

ThEOREM 6.1. (i) If $\Delta^{\prime}$ is a closed, irredundant subset of $\Omega$, then

$$
\bigcup_{\mu \in \Delta^{\prime}}(\widehat{\Phi(\mu)})
$$

is a class of $R$-modules closed under direct limits and submodules formation.

(ii) Every class of $R$-modules closed under direct limit and submodule formation can be obtained in this manner. $\Delta^{\prime}$ is uniquely determined by the class.

\section{REFERENCES}

1. L. Fuchs, Infinite Abelian Groups, I, Academic Press, 1970.

2. I. Kaplansky, Modules over Dedekind rings and valuation rings, Trans. Amer. Math. Soc., 72 (1952), 327-340.

Received December 31, 1970.

The University of Iowa 



\section{PACIFIC JOURNAL OF MATHEMATICS}

\section{EDITORS}

RICHARD ARENS (Managing Editor)

University of California

Los Angeles, California 90024

\section{R. A. Beaumont \\ University of Washington \\ Seattle, Washington 98105}

\section{J. DugundjI*}

Department of Mathematics University of Southern California Los Angeles, California 90007

D. Gilbarg and J. Milgram

Stanford University

Stanford, California 94305

\section{ASSOCIATE EDITORS}
E. F. BeCKenBaCH
B. H. NeumanN
F. WOLF
K. YoSHIDA

\section{SUPPORTING INSTITUTIONS}

\author{
UNIVERSITY OF BRITISH COLUMBIA \\ CALIFORNIA INSTITUTE OF TECHNOLOGY \\ UNIVERSITY OF CALIFORNIA \\ MONTANA STATE UNIVERSITY \\ UNIVERSITY OF NEVADA \\ NEW MEXICO STATE UNIVERSITY \\ OREGON STATE UNIVERSITY \\ UNIVERSITY OF OREGON \\ OSAKA UNIVERSITY
}

\author{
UNIVERSITY OF SOUTHERN CALIFORNIA \\ STANFORD UNIVERSITY \\ UNIVERSITY OF TOKYO \\ UNIVERSITY OF UTAH \\ WASHINGTON STATE UNIVERSITY \\ UNIVERSITY OF WASHINGTON

$* * *$
$*$
AMERICAN MATHEMATICAL SOCIETY
NAVAL WEAPONS CENTER

The Supporting Institutions listed above contribute to the cost of publication of this Journal, but they are not owners or publishers and have no responsibility for its content or policies.

Mathematical papers intended for publication in the Pacific Journal of Mathematics should be in typed form or offset-reproduced, (not dittoed), double spaced with large margins. Underline Greek letters in red, German in green, and script in blue. The first paragraph or two must be capable of being used separately as a synopsis of the entire paper. Items of the bibliography should not be cited there unless absolutely necessary, in which case they must be identified by author and Journal, rather than by item number. Manuscripts, in duplicate if possible, may be sent to any one of the four editors. Please classify according to the scheme of Math. Rev. Index to Vol. 39. All other communications to the editors should be addressed to the managing editor, or Elaine Barth, University of California, Los Angeles, California, 90024.

100 reprints are provided free for each article, only if page charges have been substantially paid. Additional copies may be obtained at cost in multiples of 50 .

The Pacific Journal of Mathematics is issued monthly as of January 1966. Regular subscription rate: $\$ 60.00$ a year (6 Vols., 12 issues). Special rate: $\$ 30.00$ a year to individual members of supporting institutions.

Subscriptions, orders for back numbers, and changes of address should be sent to Pacific Journal of Mathematics, 103 Highland Boulevard, Berkeley, California, 94708.

PUBLISHED BY PACIFIC JOURNAL OF MATHEMATICS, A NON-PROFIT CORPORATION

Printed at Kokusai Bunken Insatsusha (International Academic Printing Co., Ltd.), 270, 3-chome Totsuka-cho, Shinjuku-ku, Tokyo 160, Japan

* C. R. DePrima California Institute of Technology, Pasadena, CA 91109, will replace J. Dugundji until August 1974. 


\section{Pacific Journal of Mathematics}

Vol. 50, No. $2 \quad$ October, 1974

Mustafa Agah Akcoglu, John Philip Huneke and Hermann Rost, A counter example to the Blum Hanson theorem in general spaces .............

Huzihiro Araki, Some properties of modular conjugation operator of von

Neumann algebras and a non-commutative Radon-Nikodym theorem

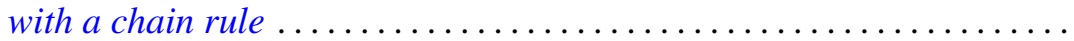

E. F. Beckenbach, Fook H. Eng and Richard Edward Tafel, Global properties of rational and logarithmico-rational minimal surfaces .....

David W. Boyd, A new class of infinite sphere packings ............. 383

K. G. Choo, Whitehead Groups of twisted free associative algebras ........

Charles Kam-Tai Chui and Milton N. Parnes, Limit sets of power series

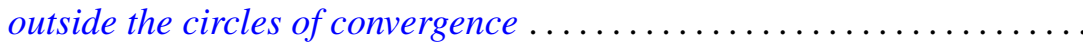

Allan Clark and John Harwood Ewing, The realization of polynomial

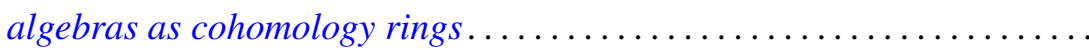

Dennis Garbanati, Classes of circulants over the p-adic and rational

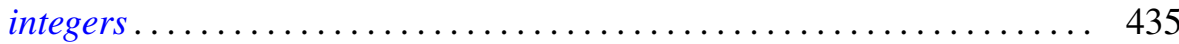

Arjun K. Gupta, On a "square" functional equation ................... 449

David James Hallenbeck and Thomas Harold MacGregor, Subordination and extreme-point theory ............................. 455

Douglas Harris, The local compactness of $v X \ldots \ldots . . . . . . . . . . . .4469$

William Emery Haver, Monotone mappings of a two-disk onto itself which fix the disk's boundary can be canonically approximated by homeomorphisms .................................. 477

Norman Peter Herzberg, On a problem of Hurwitz .................. 485

Chin-Shui Hsu, A class of Abelian groups closed under direct limits and subgroups formation ............................... 495

Bjarni Jónsson and Thomas Paul Whaley, Congruence relations and multiplicity types of algebras.....................

Lowell Duane Loveland, Vertically countable spheres and their wild sets.

Nimrod Megiddo, Kernels of compound games with simple components ....

Russell L. Merris, An identity for matrix functions ........ . .

E. O. Milton, Fourier transforms of odd and even tempered distributions ...

Dix Hayes Pettey, One-one-mappings onto locally connected generalized continua

Mark Bernard Ramras, Orders with finite global dimension

Doron Ravdin, Various types of local homogeneity. .

George Michael Reed, On metrizability of complete Moore spaces ...

Charles Small, Normal bases for quadratic extensions ..

Philip C. Tonne, Polynomials and Hausdorff matrices.... . . 